\title{
Escala de Otimismo para Adultos: Construção e Validação
}

\author{
Márcia Calixto dos Santos ${ }^{1}$ \\ Solange Muglia Wechsler ${ }^{2}$ \\ ${ }^{1}$ Centro Universitário Salesiano de São Paulo \\ ${ }^{2}$ Pontifícia Universidade Católica de Campinas
}

\begin{abstract}
Resumo
O otimismo consiste em expectativas positivas quanto ao futuro e persistência diante das adversidades. Devido às lacunas teóricas referentes à sua avaliação, no âmbito nacional, esta pesquisa objetivou construir uma escala de otimismo para adultos e investigar suas propriedades psicométricas. Participaram 555 adultos, 18 a 77 anos $(M=33,6 ; D P=11,5)$, sendo a maioria mulheres $(65,4 \%)$. Após a construção dos itens, foram realizadas análise semântica, análise de juízes, análise fatorial exploratória e verificou-se a precisão. A análise semântica e a análise de juízes indicaram evidências de validade baseadas no conteúdo. A análise fatorial exploratória indicou um modelo de dois fatores, que explicou 47,3\% da variância dos dados. Os valores de alfa de Cronbach indicaram a precisão do instrumento $(\alpha=0,94$ - Fator 1 e $\alpha=0,92$ - Fator 2). Concluiu-se que a escala proposta apresenta evidências de validade baseadas no conteúdo, na estrutura interna e precisão.

Palavras-chave: otimismo, psicologia positiva, saúde mental, avaliação psicológica
\end{abstract}

Optimism Scale for adults: Construction and Validation

\begin{abstract}
Optimism consists of positive expectations about the future and persistence in the face of adversities. Due to the theoretical gaps regarding its evaluation at the national level, this research aimed to develop an optimism scale for adults and investigated their psychometric properties. The participants were 555 adults, aged from 18 to 77 years $(M=33.6 ; S D=11.5)$, most of them women $(65.4 \%)$. After the development of the scale items, the semantic analysis, the analysis by the judges, and the exploratory factorial analysis were performed and reliability was verified. The semantic analysis and the analysis by the judges indicated evidence of validity based on content. The exploratory factorial analysis indicated a two-factor solution, which explained $47.3 \%$ of data variance. Cronbach's alpha values indicated the reliability of the instrument $(\alpha=0.94$ - Factor 1 and $\alpha=0.92$ - Factor 2). It was concluded that the proposed scale presents evidence of validity based on content, internal structure, and reliability. Keywords: optimism; positive psychology; mental health; psychological assessment
\end{abstract}

\section{Escala de Optimismo para Adultos: Construcción y Validación}

\begin{abstract}
Resumen
El optimismo consiste en expectativas positivas con respecto a futuro y persistencia ante las adversidades. Debido a omisiones teóricas referentes a evaluación en el ámbito nacional, esta investigación tuvo por objetivo construir una escala de optimismo para adultos e investigar sus propiedades psicométricas. Participaron 555 adultos, 18 a 77 años $(\mathrm{M}=33,6$, DP $=11,5)$, siendo la mayoría mujeres $(65,4 \%)$. Después de la construcción de los ítems, fue realizado análisis semántico, análisis de jueces, análisis factorial exploratorio y fue verificada la precisión. El análisis semántico y el análisis de jueces indicaron evidencias de validez basados en el contenido. El análisis factorial exploratorio indicó un modelo de dos factores, que explicó 47,3 \% de la varianza de los datos. Los valores de alfa de Cronbach indicaron la precisión del instrumento $(\alpha=0,94$ - Factor 1 y $\alpha=0,92$ - Factor 2). Se concluye que la escala propuesta presenta evidencias de validez basadas en el contenido,y en la estructura interna y precisión. Palabras clave: optimismo; psicología positiva; salud mental; evaluación psicológica
\end{abstract}

\section{Introdução}

As definições teóricas sobre o otimismo são relacionadas às expectativas das pessoas quanto ao futuro, o que implica em acreditar que os acontecimentos serão positivos (Carver \& Scheier, 2014). O otimismo corresponde a uma das características estudadas pela Psicologia Positiva (Gruman, Lumley, \& González-Morales, 2018), que consiste em uma vertente teórica voltada para o desenvolvimento de pesquisas e intervenções quanto às potencialidades e virtudes humanas (Machado, Gurgel, \& Reppold, 2017).
Mesmo quando ocorrem dificuldades e obstáculos, as pessoas com tendência ao otimismo continuam acreditando que os seus objetivos serão atingidos e mantêm os seus esforços para alcançá-los (Carver, Scheier, \& Segerstrom, 2010). Nesse sentido, considera-se que o otimismo influencia diferenças significativas entre as pessoas quanto ao bem-estar subjetivo e à forma como buscam os seus objetivos. Consequentemente, essa característica psicológica define um padrão de resposta focado no enfrentamento das adversidades, sem que a pessoa evite o contato com os sentimentos negativos delas decorrentes (Segerstrom, Carver, 
\& Scheier, 2017). Além disso, o otimismo também é considerado como uma disposição da pessoa, de certa forma, estável no que se refere às situações de vida (Ottati \& Noronha, 2017).

As pesquisas têm evidenciado a importância do otimismo com relação a melhores resultados em saúde física e mental (Conversano et al., 2010), como, por exemplo, no tratamento do câncer de mama (Bastianello \& Hutz, 2016); na adesão de pacientes a programas de tratamento de saúde em geral (Geers, Wellman, Seligman, Wuyek, \& Neff, 2010); em níveis reduzidos de sintomas psicopatológicos (Hernández, Martín-Brufau, Carrillo, Berna, \& Gras, 2010). Considera-se que altos níveis de otimismo podem influenciar a tendência da pessoa a adotar um comportamento de enfrentamento proativo com relação à sua saúde (Chang \& Chan, 2015). Em outros contextos, pesquisas científicas também têm demonstrado a importância do otimismo, por exemplo, no trabalho (Carlomagno, Natividade, Oliveira, \& Hutz, 2014; Kluemper, Little, \& Degroot, 2009), na educação (Krypel \& Henderson-King, 2010; Eicher, Staerklé, \& Clémence, 2014) e nos relacionamentos interpessoais (Neff \& Geers, 2013).

Existem três teorias principais sobre o otimismo, que correspondem ao estilo explicativo (Forgeard \& Seligman, 2012), ao otimismo disposicional (Carver \& Scheier, 2002) e ao otimismo fundado (Palenzuela, 1987). A teoria do estilo explicativo refere-se à maneira como o indivíduo explica as causas dos acontecimentos negativos ou positivos (Seligman, 2012). Essa teoria envolve três dimensões: a interna-externa, segundo a qual as causas dos eventos são atribuídas à pessoa ou à situação; a estável-instável, que significa que as causas dos acontecimentos são duradouras ou limitadas no tempo e a global-específica, a qual explica que as causas dos eventos interferirão em situações diversas ou restritas (Kamen \& Seligman, 1987; Roepke \& Seligman, 2016).

Quanto à teoria do otimismo disposicional, está baseada em expectativas generalizadas e relativamente estáveis de que os resultados futuros serão positivos (Carver \& Scheier, 2014). De acordo com essa visão, a pessoa com tendência ao otimismo apresenta confiança quanto à possibilidade de atingir os seus objetivos, o que influencia diretamente a sua persistência, mesmo diante de dificuldades significativas (Gavrilov-Jerkovic, Jovanovic, Zuljevic, \& Brdaric, 2014).

Por fim, o conceito de otimismo fundado se refere a uma fundamentação realista na qual as pessoas se baseiam para enfrentar as dificuldades da vida, sendo relacionada a expectativas de controle quanto ao êxito, à autoeficácia e ao locus de controle interno (Contador et al., 2013). Mais especificamente, a autoeficácia corresponde à crença nas próprias capacidades para lidar com as adversidades ou alcançar um objetivo e o locus de controle interno relaciona-se à percepção da pessoa de que os acontecimentos e conquistas dependem de suas próprias ações. Assim, essa teoria situa o otimismo em uma perspectiva mais realista e abrangente (Contador, Fernandéz-Calvo, Palenzuela, Miguéis, \& Ramos, 2012).

Essas três teorias são complementares, entretanto apresentam algumas diferenças relevantes. Enquanto a teoria do otimismo disposicional está alicerçada nas expectativas positivas generalizadas quanto ao futuro (Bastianello \& Hutz, 2015), a teoria do estilo explicativo preocupa-se em compreender de que forma as interpretações da pessoa sobre os acontecimentos passados influenciará as suas expectativas e os seus comportamentos quanto às diferentes situações de vida (Lee, Wu, \& Lee, 2016). Por outro lado, a teoria do otimismo fundado amplia o conceito de expectativas de controle, articulando expectativas de êxito, de autoeficácia e de locus de controle, evidenciando o impacto que causam na busca de objetivos e no enfrentamento de adversidades (Palenzuela, 2014).

Com relação à avaliação de otimismo em adultos no Brasil, foi adaptado e validado o Life Orientation Test Revised - LOT-R (Bastianello, Pacico, \& Hutz, 2014), baseado na teoria do otimismo disposicional (Scheier, Carver, \& Bridges, 1994). Esse instrumento é composto por dez itens que avaliam otimismo e pessimismo (por sua vez, compreendido como uma tendência contrária ao otimismo), como pólos opostos de um continuum, sendo quatro itens considerados apenas como distratores (Chiesi, Galli, Primi, Borgi, \& Bonacchi, 2013). Ressalta-se que é um instrumento breve, indicado para levantar informações quanto a tendências da pessoa ao otimismo ou pessimismo, e para ser utilizado em pesquisas, porém não é apropriado para realizar diagnósticos psicológicos mais complexos (Hutz, 2014).

Além disso, as publicações científicas nacionais sobre otimismo e sua avaliação ainda são restritas no Brasil (Santos \& Wechsler, 2015), especialmente considerando a construção de um instrumento, especificamente, voltado para adultos. De modo geral, quanto à Psicologia Positiva, no âmbito nacional, ressalta-se a importância de ampliar os temas de investigação e os estudos de construção de instrumentos baseados na realidade sociocultural do país (Pires, Nunes, \& Nunes, 2015). Assim, diante desse cenário, esta pesquisa 
objetivou construir e investigar as propriedades psicométricas de uma Escala de Otimismo para Adultos.

\section{Método}

Esta pesquisa foi aprovada pelo Comitê de Ética em Pesquisa com Seres Humanos com o parecer CAAE: 60507516.1.0000.5481. Foram realizados dois estudos para verificar as propriedades psicométricas da Escala de Otimismo para Adultos, sendo que o primeiro foi composto por três etapas apresentadas na sequência:

\section{Estudo 1. Construção dos Itens, Análise Semântica e} Análise de Juízes

Inicialmente, foram construídos 90 itens para compor a Escala de Otimismo para Adultos, a qual corresponde a um instrumento de autorrelato, desenvolvido com base nas teorias do Otimismo Disposicional (Carver \& Scheier, 2002), do Estilo Explicativo (Seligman, 1998) e do Otimismo Fundado (Palenzuela, 1987). Assim, a construção dos itens envolveu conceitos relacionados a expectativas de futuro, estilo explicativo, autoeficácia e persistência, considerando uma perspectiva mais abrangente do construto otimismo. Ressalta-se que os itens foram divididos em três fatores: Expectativas (positivas e negativas), Autoeficácia e Persistência.

Posteriormente, foi realizada a análise semântica para verificar se uma amostra da população-alvo, com nível de escolaridade restrito ao ensino fundamental, compreenderia os itens da versão inicial da Escala de Otimismo para Adultos, conforme o esperado.

\section{Participantes}

Participaram deste estudo quatro adultos, dois homens e duas mulheres, com idade média de 57 anos $(D P=4)$ e nível de escolaridade máximo correspondente ao ensino fundamental. Essa amostra foi selecionada por conveniência.

\section{Instrumento}

Foi utilizada a Escala de Otimismo para Adultos (versão inicial), composta por 90 itens, divididos em três fatores relacionados ao otimismo: Expectativas (positivas e negativas), Autoeficácia e Persistência. Destaca-se que a escala foi composta por itens positivos e negativos.

\section{Procedimento}

Os participantes foram convidados para participar do estudo e, mediante a anuência, assinaram o
Termo de Consentimento Livre e Esclarecido (TCLE). $\mathrm{Na}$ sequência, de forma individual, foi apresentado ao participante o conceito de otimismo e verificado se compreenderia os itens da escala conforme o esperado. Os itens não compreendidos foram reformulados e reapresentados ao participante, buscando atingir sua inteligibilidade.

\section{Resultados}

A análise semântica indicou que a maioria dos itens foi compreendida pelos participantes da forma esperada. Assim, apenas 4,44\% dos 90 itens propostos não foram compreendidos devidamente, sendo submetidos a uma nova apreciação. Na segunda análise realizada, verificou-se a compreensão adequada dos participantes quanto aos sentidos atribuídos aos quatro itens reformulados, que foram retidos para a composição da escala. Posteriormente, foi realizada a análise de juízes com o objetivo de verificar a representatividade de cada item proposto para a Escala de Otimismo para Adultos.

\section{Participantes}

Participaram como juízes independentes cinco especialistas em Psicologia, do sexo feminino, com experiência em Avaliação Psicológica. Como critérios de inclusão, as participantes deveriam ter experiência na área de construção de instrumentos psicológicos, conhecimentos sobre otimismo, disponibilidade para realizar a análise e assinar o Termo de Consentimento Livre e Esclarecido (TCLE). A amostra foi selecionada por conveniência.

\section{Instrumento}

Para essa etapa do estudo, foi desenvolvido um formulário específico para facilitar a compreensão e a objetividade na análise dos juízes. Nesse formulário, constavam os objetivos da pesquisa, a definição teórica sobre o construto otimismo, a descrição dos respectivos fatores propostos e uma tabela com os itens elaborados para compor a Escala de Otimismo para Adultos.

\section{Procedimento}

Após aceitar participar do estudo e assinar o TCLE (conforme resolução 466/2012 do Conselho Nacional de Saúde), cada juiz foi orientado, individualmente, via e-mail. Assim, os juízes receberam o material teórico com as instruções para analisar os itens elaborados para a escala. Visando verificar a 
representatividade dos itens, nesse material, para cada item havia um espaço em branco, de maneira que o juiz deveria identificar a qual fator o item se referia, conforme recomendado por Pasquali (2013).

\section{Análise de Dados}

Foi realizada a tabulação referente à frequência e porcentagem de concordância das respostas dos juízes para cada item da escala. Os itens que apresentaram índice de concordância (IC) de 80\% ou mais, considerada quase perfeita, entre as análises dos juízes, foram retidos para a composição da escala. Estabeleceu-se como critério que aqueles que não atingissem esse valor, seriam revistos e submetidos a uma nova avaliação, até que se obtivesse o índice mínimo de concordância estabelecido (80\%), conforme indicado por Pasquali (2016).

\section{Resultados}

Foram necessárias três fases de avaliação por juízes. A Tabela 1 indica a porcentagem de concordância entre os juízes para cada item analisado, obtida em cada uma das fases de avaliação realizadas.
Na primeira fase da avaliação de juízes, 48 itens obtiveram índice de concordância (IC) igual a 100\%, considerada quase perfeita, sendo que 18 itens referiam-se a Expectativas, 12 à Autoeficácia e 18 à Persistência. Além disso, 25 itens obtiveram IC de 80\%, correspondendo a: oito itens para avaliar Expectativas, sete para medir Autoeficácia e 10 para avaliar Persistência. Assim, 73 itens apresentaram concordância considerada quase perfeita (Pasquali, 1999). Foram identificados 11 itens com IC de 60\%, considerada concordância substancial, porém devido ao critério inicial estabelecido pelas pesquisadoras (IC $\geq 80 \%$ ), foram reformulados para uma segunda avaliação. Por fim, optou-se por excluir sete itens com IC igual a $40 \%$ e $20 \%$, por corresponderem à concordância moderada e baixa, respectivamente. Mais especificamente, entre os itens excluídos com IC igual a 40\%, um se referia a Expectativas, três à Autoeficácia e um à Persistência. Entre os itens excluídos com IC de 20\%, dois relacionavam-se à Autoeficácia.

$\mathrm{Na}$ segunda fase da avaliação de juízes, dois itens obtiveram IC de 100\%, sendo um relacionado à Autoeficácia e o outro à Persistência e, ainda, três itens obtiveram IC de 80\%, sendo relacionados a Expectativas. Assim, cinco itens apresentaram concordância quase

Tabela 1.

Indice de Concordância entre Juizes para os Itens da Escala

\begin{tabular}{ll}
\hline Avaliação 1 & \\
\hline Índice de concordância (IC) & Itens da escala \\
\hline $80-100 \%$ & $1,2,3,4,5,6,10,11,12,13,14,15,16,17,19,20,21$, \\
& $22,23,25,26,27,28,29,30,31,32,33,35,36,38,39$, \\
& $41,42,43,44,45,46,47,48,49,50,52,53,55,56,57$, \\
& $58,59,60,62,63,64,65,66,67,68,71,72,73,74,75$, \\
& $76,77,80,82,83,85,86,87,88,89,90$ \\
$60-80 \%$ & $8,18,24,37,51,54,69,70,78,84$ \\
$40-60 \%$ & $7,40,61,79,81$ \\
$20-40 \%$ & 9,34 \\
\hline Avaliação 2 & \\
\hline Índice de concordância (IC) & Itens da escala \\
$80-100 \%$ & $18,51,54,69,84$ \\
$60-80 \%$ & 78 \\
$40-60 \%$ & $8,24,37$ \\
$20-40 \%$ & 70 \\
\hline Avaliação 3 & \\
\hline Índice de concordância (IC) & Itens da escala \\
$80-100 \%$ & 78 \\
\hline
\end{tabular}


perfeita e foram considerados válidos para a escala. No entanto, três itens correspondentes à Autoeficácia obtiveram IC de $40 \%$, ou seja, concordância moderada, e um item, também referente a esse fator, apresentou IC de $20 \%$, considerada concordância baixa. Dessa forma, optou-se por excluí-los nessa fase de avaliação. Apenas o item 78 obteve IC de $60 \%$ e foi reformulado para uma nova análise. Assim, foi necessária uma terceira avaliação para este único item, relacionado à Autoeficácia, o qual obteve IC de 100\%, sendo considerado válido para a composição da escala.

Por fim, 79 itens foram classificados como válidos para compor a versão piloto da Escala de Otimismo para Adultos, apresentando IC $\geq 80 \%$, o que indica evidências de validade baseadas no conteúdo. Dessa maneira, dentre os 79 itens retidos, 29 correspondiam a Expectativas, 21 a Autoeficácia e 29 a Persistência.

\section{Estudo 2. Evidências de Validade Baseadas na}

\section{Estrutura Interna e Precisão}

Este estudo objetivou verificar evidências de validade baseadas na estrutura interna. Para tanto, foram realizadas a análise fatorial exploratória (AFE) e verificadas estimativas de precisão da Escala de Otimismo para Adultos.

\section{Participantes}

Participaram 555 adultos, selecionados por conveniência, com idades entre 18 e 77 anos $(M=33,6 ; D P=$ 11,5). A maioria dos participantes era mulheres $(65,4 \%$; $N=363)$, concluiu ou estava cursando o nível superior $(55,7 \% ; N=309)$ e era solteira $(46,5 \% ; N=258)$.

\section{Instrumentos}

Foi utilizada uma ficha de identificação para obter informações sobre idade, sexo, nível de escolaridade e estado civil do participante. Além disso, foi aplicada a Escala de Otimismo para Adultos, instrumento de autorrelato com 79 itens, desenvolvidos a partir da análise semântica e análise de juízes. Os itens eram respondidos com base em uma escala do tipo Likert de cinco pontos, variando entre Nem um pouco a Extremamente. Assim, a pontuação da escala foi organizada da seguinte maneira: (1) Nem um pouco; (2) Um pouco; (3) Moderadamente; (4) Bastante e (5) Extremamente.

\section{Procedimento}

A coleta de dados foi realizada on-line e presencial. Assim, mediante a autorização de uma instituição particular de ensino superior, do interior de São Paulo, foram convidados universitários para participar da pesquisa, sendo esclarecidos os respectivos objetivos e procedimentos. Aqueles que aceitaram participar assinaram o TCLE, em duas vias, sendo que uma delas lhes foi entregue e a outra arquivada por uma das pesquisadoras. A coleta de dados foi realizada, de forma coletiva e em sala de aula, em um único encontro, com duração aproximada de 30 minutos. Foram excluídos os participantes que não responderam a todos os itens dos instrumentos aplicados, além de dois participantes com idade abaixo de 18 anos.

$\mathrm{Na}$ sequência, os universitários foram solicitados a indicar familiares para participarem do estudo. A partir da aceitação do familiar indicado pelos universitários, foi enviado um link do Surveymonkey (software para realização de pesquisas), via rede social ou e-mail, que direcionava o participante às informações do estudo. Esse link apresentava os objetivos da pesquisa, o TCLE, ao final do qual o participante deveria emitir o aceite eletrônico quanto à sua participação. Somente após a leitura e aceite do TCLE, o participante era direcionado para as questões da pesquisa. A duração do processo de resposta on-line ocorreu entre 20 e 30 minutos.

\section{Análise de Dados}

Foi realizada a análise fatorial exploratória (AFE), que é um conjunto de técnicas multivariadas destinadas a investigar a estrutura subjacente em uma determinada matriz de dados (Brown, 2006). Ressalta-se que as decisões tomadas pelo pesquisador, ao realizar a AFE, devem ser baseadas em critérios teóricos e metodológicos objetivos, a fim de garantir a obtenção de modelos fatoriais adequados (Damásio, 2012). Antes de proceder a AFE, verificou-se se os dados poderiam ser submetidos à fatoração, sendo realizada a Medida Kaiser-Meyer-Olkin (KMO), que verifica se há um número satisfatório de correlações significativas entre os itens, e o Teste de Esfericidade de Bartlett, que verifica a significância das correlações em uma matriz de dados (Hair, Anderson, Tatham, \& Black, 2005).

$\mathrm{Na}$ execução da AFE, uma decisão importante refere-se à definição do número de fatores a ser retido (Damásio, 2012). Assim, foi utilizado o Scree Plot para observar o gráfico dos eigenvalues, verificando quais fatores apresentavam maiores eingevalues e, portanto, eram responsáveis por uma maior variância explicada. Além disso, foi utilizado o método das análises paralelas (AP), baseado no Minimum Rank Factor Analysis (Timmerman \& Lorenzo-Seva, 2011), que consiste na construção aleatória de um conjunto hipotético de matrizes de 
correlação de variáveis, com base na mesma dimensionalidade do conjunto de dados reais (Laros, 2004).

$\mathrm{Na}$ sequência, a solução fatorial foi verificada por meio do método de estimação Unweighted Least Squares (ULS), baseando-se em matrizes de correlação policóricas, método adequado a variáveis de nível ordinal (ex., escala Likert). Para realizar as análises, foi utilizado o software estatístico Factor, versão 10.3 (Lorenzo-Seva \& Ferrando, 2013). Foram também investigadas estimativas de precisão de cada subescala da Escala de Otimismo para Adultos, por meio do coeficiente alfa de Cronbach, cujos valores acima de 0,70 são considerados

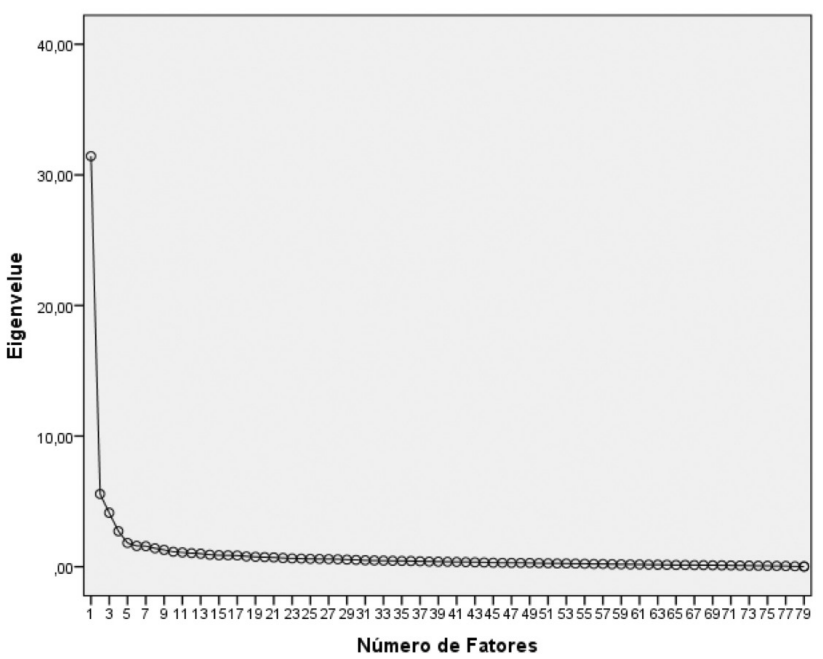

Figura 1. Scree Plot (valores próprios estimados por meio de matriz de correlação policórica). indicadores adequados de precisão do instrumento (George \& Mallery, 2002).

\section{Resultados}

O Teste de Esfericidade de Bartlett $=(g l=3081 ;$ 28635.1, $p<0,001)$ e a medida de adequação da amostra de Kaiser-Meyer-Olkin $(\mathrm{KMO})=0,970$ indicaram correlações suficientes entre as variáveis para a realização das análises pretendidas, apresentando valores considerados adequados. Em seguida, foi realizado o Scree Plot, considerando valores próprios estimados pela análise policórica, cujos resultados podem ser verificados na Figura 1.

Os resultados do Scree Plot, apresentados na Figura 1, indicaram a pertinência de uma solução de quatro fatores. Porém, ao analisar o agrupamento dos itens nesses fatores, com base na fundamentação teórica da pesquisa, observou-se que essa solução não era a mais adequada. Posteriormente, os dados foram submetidos à análise paralela (AP), cujos resultados podem ser verificados na Tabela 2 , na qual são observadas a porcentagem de variância explicada dos fatores estimados a partir dos dados reais, a porcentagem de variância explicada média estimada por meio dos dados aleatórios e a porcentagem de variância explicada alocada no percentil 95 .

Os resultados da AP também indicaram a pertinência da retenção de quatro fatores, uma vez que, a partir do quinto fator, a variância média dos dados hipotéticos foi maior que a variância dos dados reais

Tabela 2.

Resultados da Análise Paralela

\begin{tabular}{cccc}
\hline Fatores & $\begin{array}{c}\text { \% Variância } \\
\text { dados reais }\end{array}$ & $\begin{array}{c}\text { \% Variância média dados } \\
\text { aleatórios }\end{array}$ & $\begin{array}{c}\text { \% Variância alocada } \\
\text { no percentil } 95\end{array}$ \\
\hline 1 & $40.2^{*}$ & 2.8 & 3.0 \\
2 & $7.1^{*}$ & 2.7 & 2.8 \\
3 & $5.3^{*}$ & 2.6 & 2.7 \\
4 & $3.5^{*}$ & 2.5 & 2.6 \\
5 & 2.3 & 2.5 & 2.6 \\
6 & 2.0 & 2.4 & 2.5 \\
7 & 2.0 & 2.4 & 2.4 \\
8 & 1.8 & 2.3 & 2.4 \\
9 & 1.6 & 2.3 & 2.3 \\
10 & 1.4 & 2.2 & 2.3 \\
\hline
\end{tabular}


(2,5\% apresentado pela média dos dados aleatórios, contra 2,3\% dados reais). Entretanto, o modelo de quatro fatores não foi considerado adequado, com base na fundamentação teórica. Por outro lado, ainda analisando os resultados da AP, observou-se que o primeiro fator explicava $40,2 \%$ da variância dos dados empíricos, opondo-se a 2,8\% da variância média dos dados aleatórios; o segundo fator explicava 7,1\% da variância dos dados empíricos, contra $2,7 \%$ da variância média dos dados aleatórios; e o terceiro fator explicava 5,3\% da variância dos dados empíricos, opondo-se a 2,6\% da variância média dos dados aleatórios. Assim, com base nesses resultados e em critérios teóricos, interpretabilidade, procedeu-se a AFE (Hair et al., 2005), baseada na matriz de correlações dos itens, por meio da determinação de uma solução de três fatores.

Entretanto, os resultados da AFE, baseada no modelo de três fatores, indicaram 11 itens complexos, ou seja, que apresentaram carga fatorial acima de 0,30, considerada apropriada (Pasquali, 2013), em mais de um fator. Mais especificamente, observou-se que os itens complexos se referiam aos fatores Autoeficácia e Persistência, o que, possivelmente, poderia ser explicado, em parte, por uma elevada correlação entre ambos, entretanto dificultava a interpretação teórica dos dados. Além disso, 30 itens se agruparam de forma contrária à fundamentação teórica, ou seja, agruparam-se em fatores diferentes do que era esperado inicialmente. Dessa maneira, esses resultados influenciaram a decisão de realizar novamente a AFE, considerando um modelo de dois fatores.

$\mathrm{Na}$ Tabela 3, são apresentados os resultados da AFE, com base na solução de dois fatores. Considerando as cargas fatoriais dos itens, observou-se que os itens 9, 13 e 54 não carregaram em nenhum fator, portanto, foram excluídos. Na sequência, optou-se por excluir os itens que apresentaram cargas fatoriais em mais de um fator, com valores positivos considerados muito próximos (itens: 20, 23, 30, 32, 38, 39, 41, 50, 51, $68,69)$ e também aqueles que apresentaram carga fatorial em um fator diferente do esperado teoricamente (itens: 11, 16, 22, 29, 35, 37, 47, 56, 57, 59, 64, 74, 75).

Dessa forma, os resultados indicaram que o Fator 1 foi composto por 30 itens (itens: 2, 3, 5, 6, 8, 12, 14, $15,17,18,21,24,26,27,33,36,42,44,45,48,53,60$, $62,63,65,67,71,73,77,79)$ com cargas fatoriais entre 0,37 e 0,97 . Esse fator agrupou itens previstos, teoricamente, para os fatores Autoeficácia e Persistência, o que influenciou a decisão de denominá-lo como Eficácia Perseverante. Alguns exemplos de itens que compõem esse fator são: Consigo superar desafios; Eu me sinto autoconfiante; Continuo interessado mesmo quando a tarefa dura meses; Consigo o que quero porque sou insistente. Por sua vez, o Fator 2, denominado Expectativas, agrupou 22 itens (itens: 1, 4, 7, 10, 19, 25, 28, $31,34,40,43,46,49,52,55,58,61,66,70,72,76,78)$ com cargas fatoriais entre 0,32 e 0,99. Alguns exemplos de itens que compõem esse fator são: Acredito que os obstáculos serão passageiros; Penso que o meu futuro será melhor em tudo; Quando eu precisar, terei amigos com quem contar; Espero que minhas dificuldades sejam superadas. Assim, a Escala de Otimismo para Adultos constituiu-se com um total de 52 itens divididos em dois fatores: Fator 1 - Eficácia Perseverante e Fator 2 - Expectativas.

Posteriormente, foi realizada análise de precisão dos fatores da escala, estimada por meio do alfa de Cronbach $(\alpha)$. Os resultados indicaram para o Fator 1 - Eficácia Perseverante o valor $\alpha=0,94$ e para o Fator 2 - Expectativas o valor $\alpha=0,92$, o que corresponde à existência de confiabilidade nas medidas, pois quanto mais próximo de 1 for o coeficiente de precisão, menos erros de medida o instrumento apresentará ao ser utilizado (Pasquali, 2013).

\section{Discussão}

Em função da relevância do otimismo em diferentes aspectos da vida, por exemplo, com relação a trabalho, relacionamentos interpessoais e saúde mental (Segerstrom et al., 2017), esta pesquisa objetivou construir e verificar evidências de validade e estimativas de precisão de uma escala de otimismo para adultos. Assim sendo, as evidências de validade verificadas neste estudo permitiram inferir que esse instrumento apresenta propriedades psicométricas adequadas, entretanto, essas ainda são análises iniciais que precisam ser incrementadas com futuras pesquisas, inclusive considerando evidências de validade baseadas em medidas externas. Destaca-se que o LOT-R - Life Orientation Test Revised, validado para a população brasileira, não é adequado para realizar diagnósticos mais complexos (Hutz, 2014), portanto, é relevante o aprimoramento dos estudos sobre a Escala de Otimismo para Adultos, a fim de desenvolvê-la como um instrumento válido para proceder a diagnósticos.

Inicialmente, a Escala de Otimismo para Adultos foi construída com base na compreensão do otimismo em uma perspectiva ampla. Dessa maneira, o construto foi considerado, não somente com relação às 
Tabela 3.

Fatores e Cargas Fatoriais dos Itens da Escala

\begin{tabular}{|c|c|c|c|c|c|}
\hline Itens & Fator 1 & Fator 2 & Itens & Fator 1 & Fator 2 \\
\hline Item1 & & 0.59 & Item41 & 0.40 & 0.37 \\
\hline Item2 & 0.37 & & Item 42 & 0.88 & \\
\hline Item3 & 0.51 & & Item 43 & -0.46 & 0.92 \\
\hline Item4 & & 0.53 & Item 44 & 0.74 & \\
\hline Item5 & 0.50 & & Item45 & 0.73 & \\
\hline Item6 & 0.74 & -0.31 & Item 46 & -0.33 & 0.99 \\
\hline Item 7 & & 0.46 & Item47 & & 0.49 \\
\hline Item8 & 0.75 & & Item 48 & 0.79 & \\
\hline Item9 & & & Item49 & & 0.81 \\
\hline Item10 & & 0.41 & Item50 & & 0.49 \\
\hline Item11 & 0.48 & & Item51 & 0.47 & 0.31 \\
\hline Item12 & 0.47 & & Item52 & -0.32 & 0.99 \\
\hline Item13 & & & Item53 & 0.53 & -0.33 \\
\hline Item14 & 0.54 & & Item54 & & \\
\hline Item15 & 0.37 & & Item55 & & 0.75 \\
\hline Item16 & 0.57 & & Item56 & 0.31 & 0.56 \\
\hline Item17 & 0.71 & & Item57 & & 0.54 \\
\hline Item18 & 0.47 & & Item58 & & 0.44 \\
\hline Item19 & & 0.71 & Item59 & 0.38 & 0.43 \\
\hline Item20 & 0.36 & 0.35 & Item60 & 0.82 & \\
\hline Item 21 & 0.97 & -0.53 & Item61 & & 0.95 \\
\hline Item22 & 0.65 & & Item62 & 0.45 & \\
\hline Item 23 & 0.31 & 0.35 & Item63 & 0.87 & \\
\hline Item24 & 0.53 & & Item64 & 0.56 & \\
\hline Item 25 & -0.36 & 0.97 & Item65 & 0.54 & \\
\hline Item 26 & 0.90 & & Item66 & & 0.79 \\
\hline Item27 & 0.93 & & Item67 & 0.92 & \\
\hline Item28 & & 0.83 & Item68 & 0.42 & 0.34 \\
\hline Item29 & & 0.63 & Item69 & 0.37 & 0.36 \\
\hline Item30 & 0.45 & 0.35 & Item70 & -0.34 & 0.95 \\
\hline Item31 & & 0.87 & Item71 & 0.48 & \\
\hline Item32 & 0.45 & 0.32 & Item72 & & 0.32 \\
\hline Item33 & 0.96 & -0.30 & Item73 & 0.54 & \\
\hline Item34 & -0.42 & 0.85 & Item74 & 0.46 & \\
\hline Item35 & & 0.37 & Item75 & 0.50 & 0.32 \\
\hline Item36 & 0.62 & & Item76 & & 0.40 \\
\hline Item37 & 0.59 & & Item77 & 0.40 & \\
\hline Item38 & 0.47 & 0.30 & Item78 & & 0.86 \\
\hline Item39 & 0.35 & 0.33 & Item79 & 0.94 & -0.39 \\
\hline Item 40 & & 0.88 & & & \\
\hline
\end{tabular}


expectativas positivas ou negativas de futuro (Carver \& Scheier, 2014), mas também quanto às características psicológicas com as quais se relaciona como a autoeficácia (Palenzuela, 1987, 2014) e a persistência (Forgeard \& Seligman, 2012). Na sequência, tanto a análise semântica quanto a de juízes indicaram que a escala apresenta evidências de validade baseadas no conteúdo. Mais especificamente, a análise semântica demonstrou que a maioria dos itens atendia ao critério de clareza e inteligibilidade (Pasquali, 2010), sendo possível hipotetizar que os problemas de compreensão quanto a alguns itens tenham ocorrido em função da presença de negação em sua formulação, o que costuma prejudicar a inteligibilidade. Observou-se também que, desde a primeira etapa da análise de juízes, houve concordância em níveis satisfatórios quanto à maioria dos itens avaliados, indicando que estes representavam adequadamente o construto otimismo, conforme critérios recomendados por Pasquali (2013).

A análise fatorial exploratória indicou evidências de validade baseadas na estrutura interna da escala. Assim, foi verificada a pertinência de um modelo de dois fatores, que explicou $47,3 \%$ da variância dos dados. Destaca-se que a decisão final quanto ao número de dois fatores foi pautada na sua adequação teórica e interpretabilidade, de acordo com Damásio (2012).

Dessa maneira, a Escala de Otimismo para Adultos configurou-se com 52 itens, divididos em dois fatores. O Fator 1 - Eficácia Perseverante, formado por 30 itens, corroborou a teoria do Otimismo Fundado (Palenzuela, 1987, 2014), portanto envolvendo expectativas de autoeficácia, êxito e locus de controle do indivíduo, influenciando suas ações e sua persistência na busca de objetivos e enfrentamento das adversidades (Segerstrom et al., 2017). Além disso, esses resultados também estão alinhados à teoria do estilo explicativo (Seligman, 2012), considerando que o otimismo envolve a persistência diante dos desafios e dificuldades. O Fator 2 - Expectativas, composto por 22 itens, envolveu principalmente a teoria do otimismo disposicional, pois está focado nas expectativas positivas ou negativas quanto ao futuro (Carver \& Scheier, 2002).

Os indicadores de precisão, verificados por meio do alfa de Cronbach, relacionados ao Fator 1 - Eficácia Perseverante e ao Fator 2 - Expectativas, confirmaram a confiabilidade do instrumento. Os resultados obtidos corroboraram Pasquali (2013), que ressalta que um coeficiente igual ou acima de 0,90 é o esperado para confirmar a fidedignidade de um instrumento de avaliação psicológica. Ademais, concluiu-se que a Escala de Otimismo para Adultos apresenta evidências de validade e precisão, porém ressalta-se a necessidade de continuidade dos estudos sobre suas propriedades psicométricas.

\section{Considerações Finais}

Esta pesquisa objetivou a construção e a busca por evidências de validade e estimativas de precisão da Escala de Otimismo para Adultos. Além disso, pretendeu-se contribuir com a ampliação das pesquisas em Psicologia Positiva, ainda restritas, sobre esse construto no Brasil, permitindo que essa característica psicológica possa ser avaliada em adultos. Ressalta-se que o otimismo é uma característica que influencia melhores resultados da pessoa com relação à saúde física e mental, ao trabalho, à educação e aos relacionamentos. Assim, a continuidade dos estudos psicométricos referentes a essa escala poderá contribuir para que profissionais que atuam em diferentes contextos e, principalmente, no âmbito da saúde, possam avaliar o otimismo, que corresponde a um fator de proteção no que se refere à adesão aos tratamentos e à adoção de comportamentos preventivos.

As análises realizadas também permitiram identificar que se trata de uma escala com índices satisfatórios de confiabilidade. Além disso, observou-se que é possível medir o otimismo de forma válida e fidedigna, em uma perspectiva mais ampla e pautada na realidade, considerando não apenas as expectativas positivas do indivíduo quanto aos acontecimentos futuros, mas também os fatores cognitivos e comportamentais envolvidos, como as crenças de autoeficácia e a persistência.

Uma das limitações verificadas nesta pesquisa referiu-se à dificuldade em obter uma amostra mais equilibrada quanto ao sexo, uma vez que houve predomínio de mulheres. $\mathrm{Na}$ avaliação de juízes, também houve dificuldade em encontrar um número maior de participantes que atendesse aos critérios de inclusão. Por fim, outro aspecto que pode ser considerado uma limitação refere-se ao fato de não ter sido aprofundado, nos estudos estatísticos, a análise de outras variáveis que podem ter influência nos níveis de otimismo, como a renda financeira, o estado civil, a escolaridade.

Sugerem-se novas pesquisas com a Escala de Otimismo para Adultos, a fim de complementar as análises psicométricas, como estudos baseados na Teoria de Resposta ao Item e na análise fatorial confirmatória. Ademais, sugere-se para estudos futuros considerarem amostras mais equilibradas quanto ao sexo, verificarem 
evidências de validade baseadas em variáveis externas, testarem a aplicação da escala em populações clínicas, podendo, dessa forma, complementar os estudos de evidências de validade.

\section{Referências}

Bastianello, M. R., \& Hutz, C. S. (2015). Do otimismo explicativo ao disposicional: A perspectiva da psicologia positiva. Psico-USF, 20(2), 237-247. doi: 10.1590/1413-82712015200205

Bastianello, M. R., \& Hutz, C. S. (2016). Otimismo e suporte social em mulheres com câncer de mama: Uma revisão sistemática. Psicologia: Teoria e Prática, 18(2), 19-33. doi: 10.15348/1980-6906/psicologia. v18n2p19-33

Bastianello, M. R., Pacico., J.C., \& Hutz, C. S. (2014). Optimism, self-esteem and personality: Adaptation and validation of the Brazilian Version Of The Revised Life Orientation Test (LOT-R). Psico-USF, 19(3), 523-53. doi: 10.1590/1413-82712014019003014

Brown, T. A. (2006). Confirmatory factor analysis for applied research. New York: The Guilford Press.

Carlomagno, L. L. L., Natividade, J. C., Oliveira, M. Z., \& Hutz, C. S. (2014). Relações entre criatividade, esperança, otimismo e desempenho profissional. Temas em Psicologia, 22(2), 497-508. doi: 10.9788/ TP2014.2-18

Carver, C. S., \& Scheier, M. F. (2002). Optimism. Em C. R. Snyder \& S. J. Lopez (Org.), Handbook of Positive Psychology (pp. 231-243). New York, NY: Oxford University Press.

Carver, C. S., Scheier, M. F., \& Segerstrom, S. G. (2010). Optimism. Clinical Psychological Review, 30, 879-889. doi: 10.1016/j.cpr.2010.01.006

Carver, C. S., \& Scheier, M. F. (2014). Dispositional optimism. Trends in Cognitive Sciences, 18(6), 293-297. doi: 10.1016/j.tics.2014.02.003

Chang Y., \& Chan, H. (2015). Optimism and proactive coping in relation to burnout among nurses. Journal of Nursing Management, 23, 401-408. doi: 10.1111/ jonm.12148

Chiesi, F., Galli, S., Primi, C., Borgi, P. I., \& Bonachi, A. (2013). The accuracy of the Life Orientation Test-Revised (LOT-R) in measuring dispositional optimism: Evidence from item response theory analyses. Journal of Personality Assessment, 95(5), 523529. doi: 10.1080/00223891.2013. 781029

Contador, I., Fernández-Calvo, B., Palenzuela, D. L., Miguéis, S., \& Ramos, F. (2012). Prediction of burden in family caregivers of patients with dementia: A perspective of optimism based on generalized expectancies of control. Aging \& Mental Health, 16(6), 675-682. doi: 10.1080/13607863.2012.684666

Contador, I., Fernández-Calvo, B., Palenzuela, D. L., Campos, F. R., Rivera-Navarro, J., \& Lucena, V. M. (2013). A control-based multidimensional approach to the role of optimism in the use of dementia day care services. American Journal of $\mathrm{Al}$ zheimer's Disease \& Other Dementias, 30(7), 686-693. doi: 10.1177/1533317513494439

Conversano, C., Rotondo, A., Lensi, E., Vista, O. D., Arpone, F., \& Reda, M. A. (2010). Optimism and its impact on mental and psysical well-being. Clinical Practice \& Epidemiology in Mental Health, 6, 25-29. doi: 10.2174/1745017901006010025

Damásio, B. F. (2012). Uso da análise fatorial exploratória em psicologia. Avaliação Psicológica, 11(2), 213-228.

Eicher, V., Staerklé, C., \& Clémence, A. (2014). I want to quit education: A longitudinal study of stress and optimism as predictors of school dropout intention. Journal of Adolescence, 37, 1021-1030. doi: 10.1016/j.adolescence.2914.07.007

Forgeard, M. J. C., \& Seligman, M. E. P. (2012). Seeing the glass half full: A review of the causes and consequences of optimism. Pratiques Psychologiques, 18(2), 107-120. doi:10.1016/j.prps.2012.02.002

Geers, A. L., Wellman, J. A., Seligman, L. D., Wuyek, L. A., \& Neff, L. A. (2010). Dispositional optimism, goals, and engagement in health treatment programs. Journal of Behavioral Medicine, 33, 123-134. doi: 10.1007/s10865-009-9238-z

George, D., \& Mallery, P. (2002). SPSS for Windows step by step: A simple guide and reference. 11.0 update (4th ed.). Boston: Allyn \& Bacon.

Gavrilov-Jerkovic, V., Jovanovic, V., Zuljevic, D., \& Brdaric, D. (2014). When less is more: A short version of the Personal Optimism Scale and the Self-Efficacy Optimism Scale. Journal of Happiness Studies, 15, 455-474. doi: 10.1007/ s10902-013-9432-0

Psico-USF, Bragança Paulista, v. 25, n. 1, p. 89-100, jan./ mar. 2020 
Gruman, J. A., Lumley, M. N., \& González-Morales, M. G. (2018). Incorporating balance: Challenges and opportunities for positive psychology. Canadian Psychology, 59(1), 54-64. doi: 10.1037/cap0000109

Hair, J. F., Anderson, R. E., Tatham, R. L., \& Black, W. C. (2005). Análise multivariada de dados. (5 ${ }^{\mathrm{a}}$ ed.). Porto Alegre: Bookman.

Hernández, O. S., Martín-Brufau, R., Carrillo, F. X. M., Berna, F. J. C., \& Gras, R. M. L. (2010). Relación entre optimismo, creatividad y sintomas psicopatológicos, en estudiantes universitários. Eletronic Journal of Research in Educational Psychology, 8(3), 1151-1178.

Hutz, C. S. (2014). Avaliação em psicologia positiva. Porto Alegre, RS: Artmed, 2014.

Kamen, L. P., \& Seligman, M. E. P. (1987). Explanatory style and health. Current Psychological Research \& Reviews, 6(3), 207-218.

Kluemper, D. H., Little, L. M., \& DeGroot, T. (2009). State or trait: Effects of state optimism on jobrelated outcomes. Journal of Organizational Behavior, 30, 209-231. doi: 10.1002/job.591

Krypel, M. N., \& Henderson-King, D. (2010). Stress, coping styles, and optimism: Are they related to meaning of education in student's lives? Social Psychology of Education, 13, 409-424. doi: 10.1007/ s11218-010-9132-0

Laros, J. A. (2004). O uso da análise fatorial: Algumas diretrizes para pesquisadores. Em L. Pasquali (Org.), Análise fatorial para pesquisadores, (pp. 163193). Petrópolis: Vozes.

Lee, Y. C., Wu, W. L., \& Lee, Y. C. (2016). Explanatory style differences in health literacy: A survey among young adults in Taiwan. Psychology, Health \& Medicine, 21(2), 189-197. doi: 10.1080/13548506.2014.994536

Lorenzo-Seva, U., \& Ferrando, P. J. (2013). FACTOR 9.2: A comprehensive program for fitting exploratory and semiconfirmatory fator analysis and IRT Models. Applied Psychological Measurement, 37(6), 497-498. doi: 10.1177/0146621613487794

Machado, F. A., Gurgel, L. G., \& Reppold, C. T. (2017). Intervenções em Psicologia Positiva na reabilitação de adultos e idosos: Revisão da literatura. Estudos de Psicologia (Campinas), 34(1), 119-130. doi: 10.1590/1982-02752017000100012
Neff, L. A., \& Geers, A. L. (2013). Optimistic expectations in early marriage: A resource or vulnerability for adaptive relationship functioning? Journal of Personality and Social Psychology, 105(1), 38-60. doi: $10.1037 /$ a0032600

Ottati, F., \& Noronha, A. P. P. (2017). Factor structure of the Life Orientation Test -

Revised (LOT-R). Acta Colombiana de Psicologia, 20(1), 32-39. doi: 10.14718/ACP.2017.20.1.3

Palenzuela, D. L. (1987). Sphere-specific measures of perceived control: Perceived contingency, perceived competence, or what? A critical evaluation of Paulhus and Christie's approach. Journal of Research in Personality, 21, 264-286. doi: 10.1016/0092-6566(87)90011-0

Palenzuela, D. L. (2014). Antecedentes y gestación del concepto de optimismo fundado. Boletim Ibero-americano de criatividade e inovação, 3, 50-52. Recuperado de http://www.criabrasilis.org.br/arquivos/pdfs/153_bcriai_tres.pdf

Pasquali, L. (1999). Instrumentos psicológicos: Manual prático de elaboração. Brasília, DF: LabPAM \& IBAP.

Pasquali, L. (2010). Testes referentes a construto: Teoria e modelo de construção. In L. Pasquali (Org.), Instrumentação psicológica: fundamentos e práticas (pp. 39-71). Porto Alegre, RS: Artmed.

Pasquali, L. (2013). Psicometria: Teoria dos testes na psicologia e na educação ( $5^{\mathrm{a}}$ ed.). Petrópolis, RJ: Vozes.

Pasquali, L. (2016). Princípios de elaboração de escalas. Em C. Gorenstein, Y. P. Wang, \& I. Hungerbühler (Orgs.), Instrumentos de avaliação em saúde mental (pp. 4-12). Porto Alegre, RS: Artmed.

Pires, J. G., Nunes, M. F. O., \& Nunes, C. H. S. S. (2015). Instrumentos baseados em Psicologia Positiva no Brasil: Uma revisão sistemática. Psico-USF, 20(2), 287-295. doi: 10.1590/1413-82712015200209

Roepke, A. M., \& Seligman, M. E. P. (2016). Depression and prospection. British Journal of Clinical Psychology, 55 (Special issue paper), 23-48. doi: 10.1111/ bjc. 1207

Santos, M. C., \& Wechsler, S. M. (2015). Análise das publicações científicas sobre otimismo em saúde no último triênio. Psicologia Argumento, 33(83), 470-482. doi: $10.7213 /$ psicol.argum.33.083.AO03 
Scheier, M. F., Carver, C. S., \& Bridges, M. W. (1994). Distinguishing optimism from neuroticism (and trait anxiety, self-mastery, and self-esteem): A reevaluation of the Life Orientation Test. Journal of Personality and Social Psychology, 67, 1063-1078.

Segerstrom, S. C., Carver, C. S., \& Scheier, M. F. (2017). Optimism. Em M. Robinson, \& M. Eid (Org.), The happy mind: Cognitive contributions to well-being, (pp. 195-212). EUA: Springer, Cham.

Seligman, M. E. P. (1998). Learned optimism: How to change your mind and your life. New York: Free Press.
Seligman, M. E. P. (2012). Aprenda a ser otimista. Rio de Janeiro, RJ: Record.

Timmerman, M. E., \& Lorenzo-Seva, U. (2011). Dimensionality assessment of ordered polytomous items with parallel analysis. Psychological Methods, 16, 209-220. doi: 10.1037/a0023353

Recebido em: 21/03/2018

Reformulado em: 20/01/2019

Aceito: $12 / 02 / 2019$

Sobre as autoras:

Márcia Calixto dos Santos é psicóloga, mestra e doutora em Psicologia pelo Programa de Pós-Graduação stricto sensu da Pontifícia Universidade Católica de Campinas, linha de pesquisa Instrumentos e Processos em Avaliação Psicológica, Especialista em Psicologia Clínica - Cognitivo Comportamental. Atualmente é coordenadora e docente do curso de Psicologia do Centro Universitário Salesiano de São Paulo - UNISAL - Unidade de Americana - SP.

ORCID: https://orcid.org/0000-0002-5243-2281

E-mail:marcia.santos@unisal.br

Solange Muglia Wechsler é psicóloga, mestra e doutora - University of Georgia, e pós-doutora - Torrance Center of Creative Studies. É docente da pós-graduação stricto sensu em Psicologia da Pontifícia Universidade Católica de Campinas e coordenadora do Laboratório de Avaliação e Medidas em Psicologia. Por fim, realiza pesquisas sobre avaliação psicológica e programas em criatividade, inteligência, estilos cognitivos, liderança, altas habilidades, temperamento e psicologia positiva.

ORCID: https://orcid.org/0000-0002-9757-9113

E-mail:wechsler@lexxa.com.br

Contato com as autoras:

Centro Universitário Salesiano de São Paulo - UNISAL, Campus Maria Auxiliadora

Av. Cillos, 3500

Americana-SP, Brasil

CEP: 13467-600 